\title{
A Bi-Isotropic Hexachiral Grid in PCB
}

\author{
Ismael Barba, Ana Grande, Ana Cristina López-Cabeceira, José Represa \\ Department of Electricity \& Electronics \\ Universidad de Valladolid \\ Valladolid, Spain
}

Corresponding author: ibarba@ee.uva.es

\begin{abstract}
A bi-isotropic chiral structure has been numerically studied. It is composed of two layers of planar, 2Dchiral, honeycomb-like, parallel grids. Electromagnetic activity (gyrotropy) is observed. That leads to a strong chiral behavior with low losses.
\end{abstract}

Keywords-electromagnetic metamaterials; chiral structures

\section{INTRODUCTION}

$\mathrm{H}$ EXAGONAL structures with six-fold symmetry (also known as "honeycombs") have been widely used in different sectors, such as aerospace, construction, packaging, etc., thanks to their special properties (mechanical, optical, thermal and also electromagnetic) [1], [2].

In 1989, Wojciechowski and Branca proposed a "tilted" honeycomb, with six-fold symmetry but no mirror symmetry in a plane (that is, chiral in a plane, but not in the 3D space), as a way to obtain a Negative Poisson's Ratio (see Fig. 1) [3]. Other implications of its geometrical chirality, in terms of mechanical properties, have been studied, like enhanced compressive strength capabilities, shear stiffness compared to classical centresymmetric honeycomb configurations, or a sinclastic curvature feature [1], [4]. In 2009, David et al. proposed their application in electromagnetic absorbers [5] and, in 2010, Kopyt et al. studied the electromagnetic behavior of such structure implemented with dielectrics (polymers), in order to check

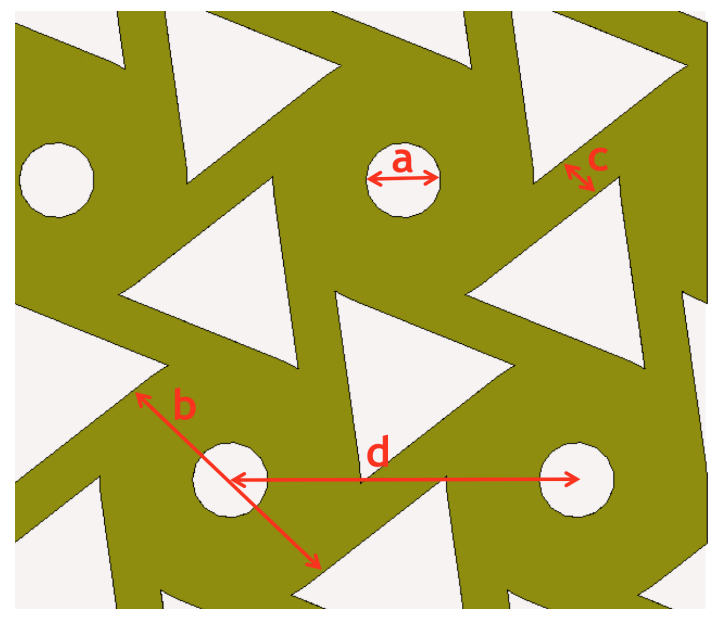

Fig. 1. A 2D-chiral structure similar to the one proposed in [3] whether their structural chirality translates into chiral electromagnetic behavior (bianisotropy) [1], with negative results. It is worth noticing that they characterized a single layer of the structure: that means the result is not geometrically chiral in the 3D space, so no bi-anisotropic behavior should have been expected [6], [7].

In this paper, we have designed and studied different implementations of a similar structure, in this case made by means of metallizations on a Printed Circuit Board (PCB); though we are also working in their experimental implementation, we are going to present here only the numeral analysis, performed by means of a commercially available simulator (CST Studio ${ }^{\mathrm{TM}}$ ). Our aim is to obtain an evidence of electromagnetic chiral behavior (gyrotropy or circular dichroism) [8]. As previously mentioned, a modification of the original, planar, structure is necessary in order to obtain a 3D chiral geometry. This may be achieved by means of a bilayer conjugated structure (easy to obtain metallizing both sides of the board) through electromagnetic coupling between the layers, if the geometry of one layer is the specular image of the other one's [9].

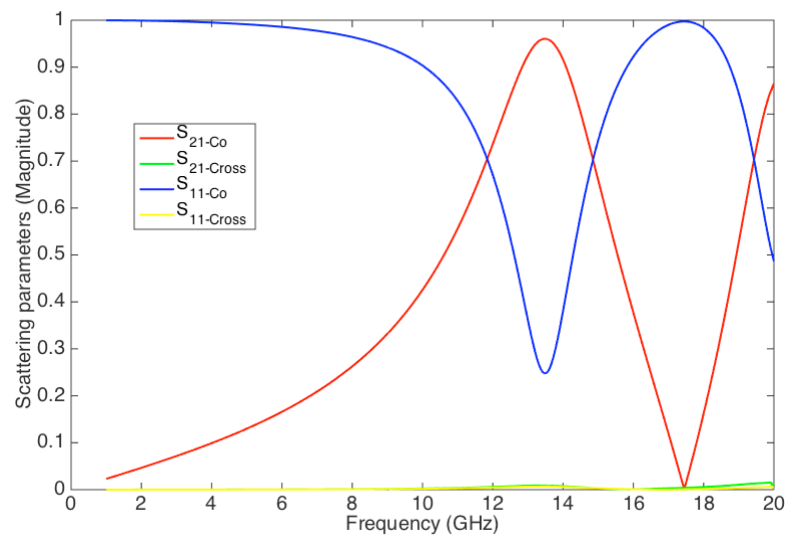

Fig. 2. Results (scattering parameters) for a plane wave normally incident on a PCB with a single metallization as shown in Fig. 1.

\section{IMPLEMENTATION AND RESULTS}

We have designed several structures using PCB technology. The basis slab is a standard Rogers RO4003C board $\left(\varepsilon_{r}=3.38\right.$, and $\operatorname{tg} \delta=0.0027$ at $10 \mathrm{GHz}$ ), 60 mils thick (i.e. $1.524 \mathrm{~mm})$. In this work, three different implementations, using one or two level metallizations have 
been considered. The geometry of each metallization is a grid as shown in Fig. 1, where $a=3.59 \mathrm{~mm}, b=12.39 \mathrm{~mm}$, $c=2.2 \mathrm{~mm}$ and $d=16.48 \mathrm{~mm}$.

In a first example, only one face of the slab is metallized. As explained in [6], [7] or [9], electromagnetic activity should be negligible. We have calculated the scattering parameters for a plane wave, normally incident on such structure, the results being shown in Fig. 2. $\mathrm{S}_{11 \text {-Co }}$ and $\mathrm{S}_{21 \text {-Co }}$ are the scattering parameters in copolarization, i.e., they represent the components of the reflected and transmitted fields that present the same polarization as the incident field. $\mathrm{S}_{11-\text { Cross }}$ and $\mathrm{S}_{21-\text { Cross }}$ are the scattering parameters in crosspolarization, i.e. the components of reflected and transmitted fields, perpendicular to the incident field. Indeed, no gyrotropy (electromagnetic activity) is observed, which is consistent, both with the theory and with the experimental results described in [1]. Also, the response of the structure shows a frequency response as expected in a "conventional" metal grid, i.e. a Faraday cage at low frequencies, with a first transmission maximum at $f=13.5$ $\mathrm{GHz}$.

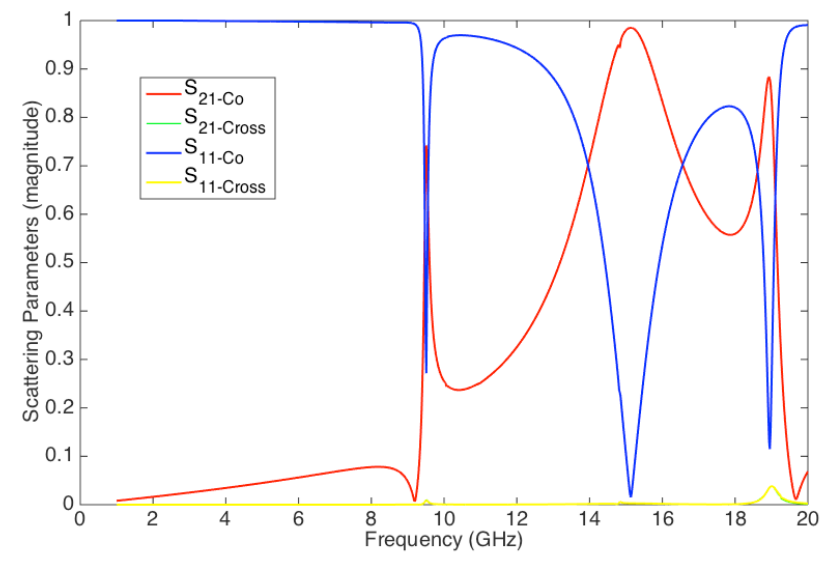

Fig. 3. Results (scattering parameters) for a plane wave normally incident on a PCB including two parallel metal layers as the one shown in Fig. 1.

In the second case, both faces of the board are metallized, the metallization on the bottom side of the board being identical to the one of the front side, i.e., the structure has mirror symmetry (no chiral). The results in this case (scattering parameters) are shown in Fig. 3. In this case, the structure presents perfect specular symmetry, so no crosspolarized parameters should be expected (i.e. $S_{11-\text { Cross }}=$ $S_{21-\text { Cross }}=0$ ). A mayor difference with the one-layer grid is the apparition of resonant transmissions, the first at $f=9.5$ $\mathrm{GHz}$, which may be attributed to the electromagnetic coupling between both metal layers.

Finally, we have modeled a third configuration: again, we have two metal layers, each in one side the PCB, their shape and dimensions being the same as in the previous examples but, in this case, one layer is the specular image of the other one (or the first one turned around), as shown in Fig. 4. That means the whole structure is reciprocal (identical, when observed from both sides) and chiral in 3D space, at least form a geometric point of view; at the same time, a magnetoelectric coupling is to be expected between the nonparallel metal arms (see Fig. 4).

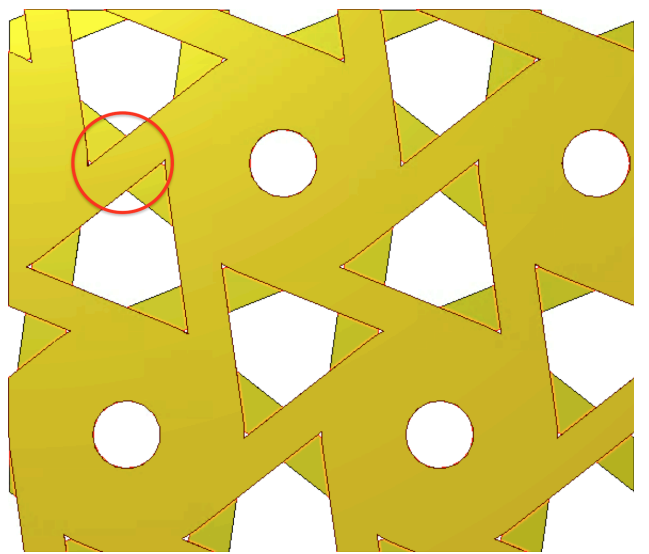

Fig. 4. A 3D-chiral structure made up of two metal layers. The dielectric slab between both has been removed in order to make the second layer visible. One of the areas of magnetoelectric coupling is highlighted by means of a red circumference.

Again, the scattering parameters for a plane wave, normally incident, are computed and shown in Fig. 5. The results show a certain resemblance with Fig. 3, the transmission maxima being placed at similar frequencies (9.5-9.3 GHz, 15.1-16.4 $\mathrm{GHz}$ and 19.0-19.1 GHz, respectively). The difference is that now, the transmission involves a rotation of the polarization plane (that is, electromagnetic activity), specially in the first resonance, where $\mathrm{S}_{11-\mathrm{Co}}$ is almost negligible, compared with $\mathrm{S}_{21-\text { Cross }}$ : that suggest, first, a rotation around $90^{\circ}$ and, second, the conservation of the linear polarization (i.e. no circular dichroism); at the same time, there is no rotation of the

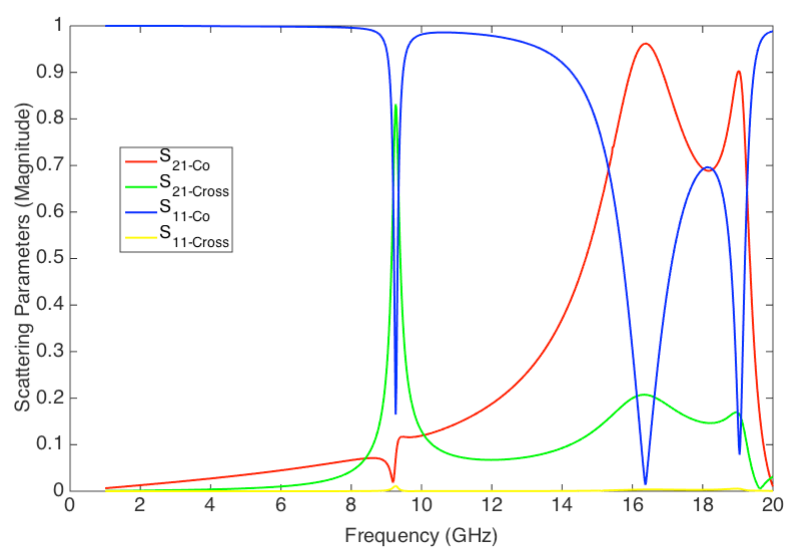

Fig. 5. Scattering Parameters for a plane wave normally incident on the bilayer 3D chiral structure pictured in Fig. 4. 
reflected wave, as is to be expected with a reciprocal structure [8].

Using the obtained scattering parameters, we have also calculated the corresponding Pasteur (chirality) parameter [8], the result being shown in Fig. 6. It follows the wellknown Condon model [8], with a resonant frequency at $f=$ $9.2 \mathrm{GHz}$ (i.e., the first transmission maximum in Fig. 5) and a negligible imaginary part, that leads to very low losses associated with the chirality. These losses have been also computed in the three cases, the energy balance (percentage of the incident energy absorbed by the structure) being represented in Fig.7. We may observe that, in the case of the bilayered chiral geometry, the energy losses are not only relatively low (a maximum of less than $28 \%$ of the incident energy in the first resonant frequency) but actually smaller than the losses of the equivalent nonchiral bilayered structure, which is consistent with the negligible value of the imaginary part of the Pasteur parameter.

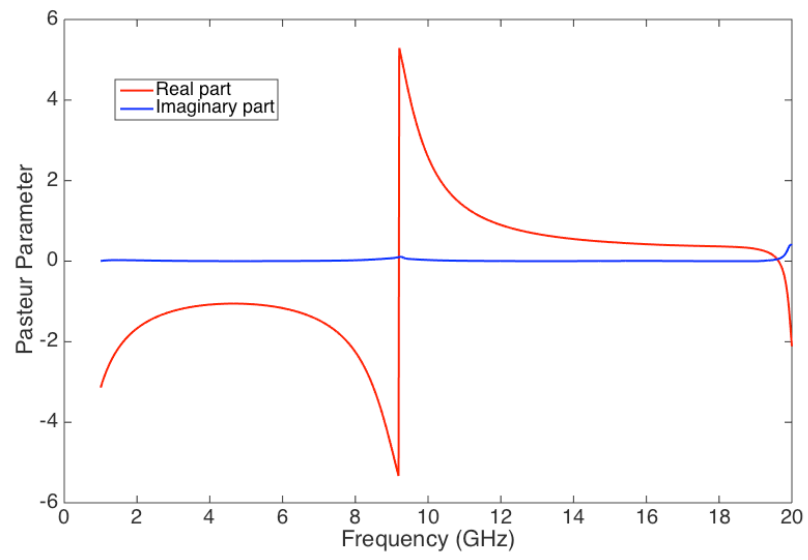

Fig. 6. Effective normalized Pasteur parameter, computed for the bilayer chiral structure pictured on Fig. 4.

\section{CONCLUSION}

We have studied the electromagnetic behavior of a hexachiral metal structure. Since the grid is two dimensional, in order to have a 3D chiral geometry we have considered a bilayered distribution, the chirality being achieved if one layer is the specular image of the other. In this case, a resonant transmission with strong electromagnetic activity, and which does not add additional energy losses is observed; that leads to a chirality parameter with a high real part and negligible imaginary part.

\section{ACKNOWLEDGEMENTS}

This work was supported in part by the Spanish Government (MINECO) and the European Commission (ERDF) through the Research Project TEC2014-55463-C32-P.

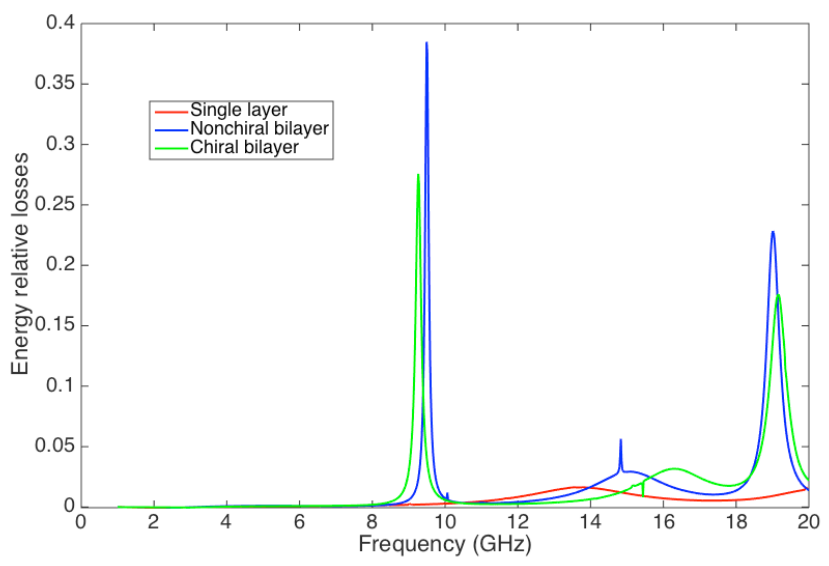

Fig. 7. Energy balance: comparison between the relative energy losses in the three studied examples.

\section{REFERENCES}

[1] P. Kopyt, R. Damian, M. Celuch, R. Ciobanu, "Dielectric properties of Chiral Honeycombs: Modelling and experiment," Composite Science and Technology, vol. 70, pp. 1080-1088, 2010.

[2] L. Seungwoo, K. Seongnam, K. Teum-Teum, K. Yushin, C. Muhan, H. L. Seung, K. Ju-Young, "Reversible Stretchable and Tunable Terahertz Metamaterials with Wrinkled Layouts," Advanced Materials, vol. 24-26, pp. 3491-3497, July 2012.

[3] K. W. Wojciechowski, A. C. Branca, "Negative Poisson ratio in a two-dimensional "isotropic" model", Physics Letters A Rev, vol. A40, pp.7222-7225, Dec. 1989.

[4] D. Bornengo, F. Scarpa, C. Remillat, "Evaluation of hexagonal chiral structure for morphing airfoil concept," Proceeding IMechE, Part G: Journal of Aerospace Engineering, vol. 219, pp. 185-192, March 2005.

[5] V. David, I. Nica, A. Salceanu, "Electromagnetic absorbers based on chiral honeycomb slab," presented at the 2009 International Symposium on Electromagnetic Compatibility - EMC Europe, Athens, Greece, June 11-12, 2009.

[6] M. Kuwata-Gonokami, N. Saito, Y. Ino, M. Kauranen, K. Jefimovs, T. Vallius, J. Turunen, Y. Svirko, "Giant Optical Activity in QuasiTwo-Dimensional Planar Nanostructures, “ Physical Review Letters, vol. 95, pp. 227401-1 - 227401-4, November 2005.

[7] I. Barba, A. C. L. Cabeceira, A.J. García-Collado, G.J. MolinaCuberos, J. Margineda, J. Represa, "Quasi-Planar Chiral Materials for Microwave Frequencies, " in Electromagnetic Waves Propagation in Complex Matter, A. Khisk, Ed: Intech, 2011, Available: http://www.intechopen.com/books/electromagnetic-wavespropagation-in-complex-matter/quasi-planar-chiral-materials-formicrowave-frequencies

[8] I. V. Lindell, A. H. Sihvola, S. A. Tretyakov, A.J. Viitanen, Electromagnetic Waves on Chiral and Bi-Isotropic Media, Artech House, 1994, MA, USA.

[9] R. Zhao, J. Zhang, J. Zhou, Th. Koschny, C. M. Soukoulis, "Conjugated gammadion chiral metamaterial with uniaxial optical activity and negative refractive index," Physical Review B, vol. 83, 030515, January 2011. 